\title{
Conductive electron heat flow along an inhomogeneous magnetic field
}

\author{
E. D. Held ${ }^{\mathrm{a})}$ \\ Utah State University, Logan, Utah 84322 \\ J. D. Callen and C. C. Hegna \\ Engineering Physics and Physics Departments, University of Wisconsin, Madison, Wisconsin 53706
}

(Received 22 May 2003; accepted 28 July 2003)

\begin{abstract}
In this work a general closure for the conductive electron heat flux parallel to an inhomogeneous magnetic field is derived and examined. Free-streaming and collisional effects are present in the drift kinetic equation which is solved using an expansion of eigenfunctions of the bounce-averaged, pitch-angle scattering operator. For bounce times short compared to transit and collision times, the subsequent heat flow closure takes the form of an integral operator acting on electron temperature variations along magnetic field lines. The general closure agrees qualitatively with previous forms for the heat flux in homogeneous magnetic geometry and importantly, predicts a substantial reduction in the heat flow due to the trapping of particles in magnetic wells and the enhanced effect of collisions near the trapped/passing particle boundary. (C) 2003 American Institute of Physics.

[DOI: $10.1063 / 1.1611883$ ]
\end{abstract}

\section{INTRODUCTION}

Recently, a theory for the conduction of electron heat along magnetic field lines that allows for arbitrary collisionality has been developed. ${ }^{1}$ This paper presents an important extension of this theory by setting forth a parallel heat flux closure scheme that allows for more general magnetic geometry. This includes the important case of helical magnetic islands growing in axisymmetric toroidal equilibria of arbitrary aspect ratio and shaping. Such a closure is relevant to numerical simulations of electron temperature evolution in magnetized plasmas where transit and collision times are long compared to bounce times. Specifically, this closure provides a quantitative means for calculating parallel electron heat transport in the presence of evolving magnetic islands or in plasmas confined by chaotic magnetic fields. The latter is of interest not only in magnetized fusion plasmas but also in astrophysical plasmas where the reduction due to charged particle trapping significantly slows electron heat transport in galaxy cluster cooling flows. ${ }^{2}$

In kinetic studies of mass and heat flow, a dominant collisional mechanism involves the scattering of a particle's parallel velocity in pitch-angle, $\zeta=v_{\|} / v$. It is the asymmetries in pitch-angle space that yield finite mass and heat flow moments. Intimately connected with the scattering of directed momentum is the variation in $|\mathbf{B}|$ that a charged particle sees as it travels along a magnetic field line. These variations act to accelerate particles parallel to field lines while to lowest order preserving their energy and magnetic moment. In strongly inhomogeneous fields, the mirror force traps a substantial fraction of particles, thereby reducing the number that can contribute to parallel heat flow. In addition, collisions between trapped and passing electrons further reduces heat flow in collisional and moderately collisional regimes. We present a general form for the parallel electron

${ }^{a)}$ Electronic mail: eheld@cc.usu.edu heat flux closure that embodies both of these effects.

In this paper, a variation of neoclassical transport theory ${ }^{3,4}$ is employed in a derivation of the parallel electron heat flow, $\mathbf{q}_{\|}$, which may be used to close the electron temperature equation. In the interest of generality, the desired closure allows for the following:

(i) multiple scale lengths parallel to the magnetic field including short scale magnetic mirroring and long scale electron temperature gradients;

(ii) general magnetic geometry allowing for helical magnetic islands embedded in axisymmetric toroidal equilibria possessing arbitrary aspect ratio and shaping;

(iii) arbitrary ordering of bounce-averaged free-streaming and pitch-angle scattering effects.

In Sec. II, a multiple-scale-length expansion along magnetic field lines is introduced and a bounce-averaged equation for the distribution function that yields the lowest-order contribution to the parallel heat flux is derived. In Sec. III the pitch-angle dependence of this equation is handled by expanding in a set of orthogonal basis functions. In Sec. IV we solve for the heat flow and in Sec. V we present an example of parallel heat flow reduction due to particle trapping and collisions in sinusoidal magnetic wells. The results are summarized in Sec. VI.

\section{ELECTRON DRIFT KINETIC EQUATION}

We begin by stating the gyroaveraged or drift kinetic equation, ${ }^{5}$

$$
\begin{aligned}
\frac{\partial f}{\partial t}+ & \left(\mathbf{v}_{\|}+\mathbf{v}_{D}\right) \cdot \nabla f+\left\{\frac{\mu}{m v} \frac{\partial B}{\partial t}+\frac{e}{m v}\left(\mathbf{v}_{\|}+\mathbf{v}_{D}\right) \cdot \mathbf{E}\right\} \frac{\partial f}{\partial v} \\
& =C(f) .
\end{aligned}
$$

Here $v$ is the speed coordinate, $\mathbf{v}_{\|}$and $\mathbf{v}_{D}$ are the parallel and perpendicular components of the guiding center motion, and 
$\mu$ is the magnetic moment. The collision operator, $C(f)$, represents binary, collisional scattering events.

For a general parallel heat flux closure, we seek an ordering scheme that is valid for general magnetic geometry. In the interest of identifying the dominant parallel freestreaming and collisional physics, we require that $\mathbf{v}_{\|} \cdot \boldsymbol{\nabla}$ $\sim C(f) \gg \mathbf{v}_{D} \cdot \boldsymbol{\nabla}$, and order the remaining terms in Eq. (1) with $\mathbf{v}_{D} \cdot \boldsymbol{\nabla}$. The result of ignoring drift, time-dependent and acceleration effects is the deceptively simple, lowest order kinetic equation,

$$
\mathbf{v}_{\|} \cdot \nabla f=C(f) .
$$

This equation captures the dominant physics of parallel electron dynamics in plasmas confined by slowly evolving, spatially inhomogeneous (with regards to the parallel direction) magnetic fields.

In order to solve Eq. (2), we introduce a multiple scale length expansion along the magnetic field by defining

$$
\Delta \sim \frac{\mathbf{v}_{\|} \cdot \nabla_{L}}{\mathbf{v}_{\|} \cdot \nabla_{l}} \ll 1
$$

This ordering takes into account the longer scale length, $L$, associated with parallel electron temperature gradients and the short mirroring scale length, $l$, set by the variations in $|\mathbf{B}|$. In terms of a helical magnetic island in a large aspect ratio tokamak, $l=q R$ and $L=q R / q^{\prime} w$ refer to the parallel scale lengths associated with the equilibrium and island, respectively; hence, $\Delta \sim l / L \sim q^{\prime} w \ll 1$. Here $q$ and $q^{\prime}$ are the safety factor and its radial derivative and $w$ is the island width. In terms of time scales, this ordering represents the ratio of the transit time across one period in $|\mathbf{B}|$ to the time needed to circumnavigate the magnetic island. For generality, we will order $C(f) \sim \mathbf{v}_{\|} \cdot \nabla_{L}$, hence the kinetic equation of interest becomes

$$
\left(\mathbf{v}_{\|} \cdot \nabla_{l}+\mathbf{v}_{\|} \cdot \nabla_{L}\right) f=C(f),
$$

which has $\mathbf{v}_{\|} \cdot \nabla_{l} \gg \mathbf{v}_{\|} \cdot \nabla_{L} \sim C(f)$.

In order to solve Eq. (4), we posit the following Chapman-Enskog-type ansatz ${ }^{6,7}$ for the distribution function:

$$
f=f_{M}+F=n\left(\frac{m}{2 \pi T}\right)^{3 / 2} e^{-\left(m v^{2} / 2 T\right)}+F,
$$

where the density, temperature, and kinetic distortion, $F$, are expanded as

$$
\begin{aligned}
& n=n^{\mathrm{eq}}+\sum_{i} \Delta^{i} n^{i}(l, L), \\
& T=T^{\mathrm{eq}}+\sum_{i} \Delta^{i} T^{i}(l, L), \\
& F=\sum_{i} \Delta^{i} F^{i}(l, L, \mathbf{v}) .
\end{aligned}
$$

Here $\mathbf{v}$ represents suitable guiding center coordinates which will be chosen later and $n^{\mathrm{eq}}$ and $T^{\mathrm{eq}}$ are spatially uniform. Writing $f_{M}^{0}$ as the combined equilibrium and lowest order perturbed Maxwellian,

$$
f_{M}^{0}=f_{M}^{\mathrm{eq}}\left[1+\frac{n^{0}}{n^{\mathrm{eq}}}-\left(\frac{3}{2}-\frac{m v^{2}}{2 T^{\mathrm{eq}}}\right) \frac{T^{0}}{T^{\mathrm{eq}}}\right],
$$

implies that $F^{0}$ and $f_{M}^{0}$ satisfy

$$
\mathbf{v}_{\|} \cdot \nabla_{L} f_{M}^{0} \sim \mathbf{v}_{\|} \cdot \nabla_{L} F^{0} .
$$

Here $f_{M}^{\mathrm{eq}}=\left(n^{\mathrm{eq}} / \pi^{3 / 2} v_{\mathrm{th}}^{3}\right) \exp \left(-v^{2} / v_{\mathrm{th}}^{2}\right)$ and the electron thermal speed, $v_{\mathrm{th}}=\sqrt{2 T^{\mathrm{eq}} / m}$. The form given for $f_{M}^{0}$ assumes that the equilibrium Maxwellian is nearly stationary such that the variable $v$ represents the random guiding center speed and the macroscopic flows satisfy $V / v_{\text {th }} \sim \Delta^{2}$. For simplicity the $M$ subscript will be dropped from the Maxwellian portion of the distribution function.

To order $\Delta^{0}$ we obtain

$$
\mathbf{v}_{\|} \cdot \nabla_{l}\left(F^{0}+f^{0}\right)=0,
$$

which has the solution

$$
F^{0}=-\left(L_{0}^{(1 / 2)} \frac{n_{e}^{0}}{n^{\mathrm{eq}}}-L_{1}^{(1 / 2)} \frac{T_{e}^{0}}{T^{\mathrm{eq}}}\right) f^{\mathrm{eq}}+g^{0}(L, \mathbf{v}),
$$

where $L_{0}^{(1 / 2)}=1$ and $L_{1}^{(1 / 2)}=3 / 2-v^{2} / v_{\text {th }}^{2}$ are Laguerre polynomials. Note that the integration constant, $g^{0}$, does not depend on $l$, the distance along a field line within a single magnetic mirror. In Chapman-Enskog theory we require that the density and temperature moments of $F^{0}$ vanish; hence

$$
n_{e}^{0}=\int d^{3} v L_{0}^{(1 / 2)} g^{0},
$$

and

$$
T_{e}^{0}=-\frac{2}{3} \frac{T^{\mathrm{eq}}}{n^{\mathrm{eq}}} \int d^{3} v L_{1}^{(1 / 2)} g^{0} .
$$

That $n_{e}^{0}$ and $T_{e}^{0}$ are independent of $l$ shows that the ordering scheme produces the intuitive result that the rapid bounce motion smooths out the lowest order perturbed density and temperature over the scale length, $l$.

To order $\Delta^{1}$ we obtain,

$$
\mathbf{v}_{\|} \cdot \nabla_{l}\left(F^{1}+f^{1}\right)+\mathbf{v}_{\|} \cdot \nabla_{L}\left(F^{0}+f^{0}\right)=C\left(F^{0}+f^{0}\right) .
$$

In accordance with neoclassical theory, we solve this equation by eliminating the electron bounce motion timescale present in the operator, $\mathbf{v}_{\|} \cdot \nabla_{l}$. Assuming a single minimum and maximum in $|B|$, we use the following averaging operators for trapped and passing particles, respectively:

$$
\oint_{-l_{b}}^{l_{b}} \frac{d l}{\left|v_{\|}\right|}
$$

and

$$
\int_{-l_{\max }}^{l_{\max }} \frac{d l}{v_{\|}}
$$

These operators annihilate the $\mathbf{v}_{\|} \cdot \nabla_{l}$ operator by integrating over closed, periodic orbits for trapped particles and open orbits for passing particles. Application of this annihilator yields

$$
\sigma\left[\mathbf{v}_{\|} \cdot \nabla_{L}\left(F^{0}+f^{0}\right)\right]=\left[C\left(F^{0}+f^{0}\right)\right],
$$


where $\sigma=1$ and 0 for passing and trapped particles, respectively, and [ ] implies a bounce-averaged quantity. Note that bounce-averaging also annihilates the $\mathbf{v}_{\|} \cdot \nabla_{L}$ for trapped electrons since they travel both parallel and antiparallel to gradients in $F^{0}$ in completing their periodic orbits. In the next two sections, the collision operator is specified and Eq. (18) is solved.

\section{COLLISION OPERATOR AND EIGENFUNCTION EXPANSION}

A dominant collisional mechanism in studies of plasma heat flow is the scattering of directed momentum due to multiple small-angle Coulomb collisions. With this in mind, we introduce the following form for the linearized collision operator:

$$
C\left(F^{0}+f^{0}\right)=C_{e, i}\left(F^{0}\right)+C_{e, e}\left(F^{0}\right),
$$

where the Lorentz scattering operator, converted from spherical velocity space to the pitch-angle-type variable, $\xi$ $\equiv \pm \sqrt{1-\mu B_{0} / E}$, is given by

$$
C_{e, i}+C_{e, e}=\bar{\nu} \frac{v_{\|}}{v \xi} \frac{\partial}{\partial \xi}\left(\frac{1-\xi^{2}}{\xi} \frac{v_{\|} B_{0}}{v B}\right) \frac{\partial}{\partial \xi} .
$$

Here $E=m v^{2} / 2$ is the electron kinetic energy and $B_{0}$ is the minimum magnetic field that an electron sees as it travels along a field line. The variable $\xi$ varies from 0 for a particle with $v_{\|}=0$ to \pm 1 for a particle with no magnetic moment. The collision frequency, $\bar{\nu}$ may approximately be written as

$$
\bar{\nu}=\nu_{\perp e i}+\nu_{\perp e e}=\frac{\nu_{e e}}{2\left(v / v_{\mathrm{th}}\right)^{3}}\left(Z_{\mathrm{eff}}+\Phi\left(\frac{v}{v_{\mathrm{th}}}\right)-G\left(\frac{v}{v_{\mathrm{th}}}\right)\right),
$$

where $Z_{\text {eff }}=\sum_{j} n_{i j} Z_{j}^{2} / \Sigma_{j} n_{i j} Z$ is summed over all ion species, $\Phi$ is the error function and $G \equiv\left(v_{\text {th }} / v\right)^{2}\left(\Phi-\left(v / v_{\text {th }}\right) \Phi^{\prime}\right) / 2$, is a function first introduced by Chandrasekhar. ${ }^{8}$ Note that although $G$ is singular at $v / v_{\text {th }}=0$, it will not compromise the behavior of the desired heat flow expression since it will appear in the form, $\exp \left[-\left(\nu_{e e} v_{\mathrm{th}}^{3} / 2 v^{4}\right)|G L|\right]$. The reference collision frequency is

$$
\nu_{e j}=4 \pi n_{j 0} e^{2} e_{j}^{2} \ln \Lambda_{e j} /\left(m_{e}^{2} v_{\mathrm{th}}^{3}\right) .
$$

Note we have assumed that the momentum restoring terms often added to $C_{e, j}$ are small. Recall that the ordering $V / v_{\text {th }} \sim \Delta^{2}$ has already been assumed; thus our pitch-angle scattering operator conserves momentum only to order $V / v_{\text {th }}$. Note also that the addition of a model energy diffusion term would improve the accuracy of the simplified, pitch-angle collision operator used in this work. Such improvement is desirable in the collisional limit where more accurate knowledge of the speed dependence of $F$ is needed to make experimental predictions.

In terms of the variable $\xi$, Eq. (18) becomes

$$
\frac{\partial}{\partial \xi} \frac{1-\xi^{2}}{\xi}\left\langle\frac{v_{\|} B_{0}}{v B}\right\rangle \frac{\partial F^{t}}{\partial \xi}=0, \quad-\xi_{t}<\xi<\xi_{t},
$$

for the trapped electron distribution, $F^{t}$, and

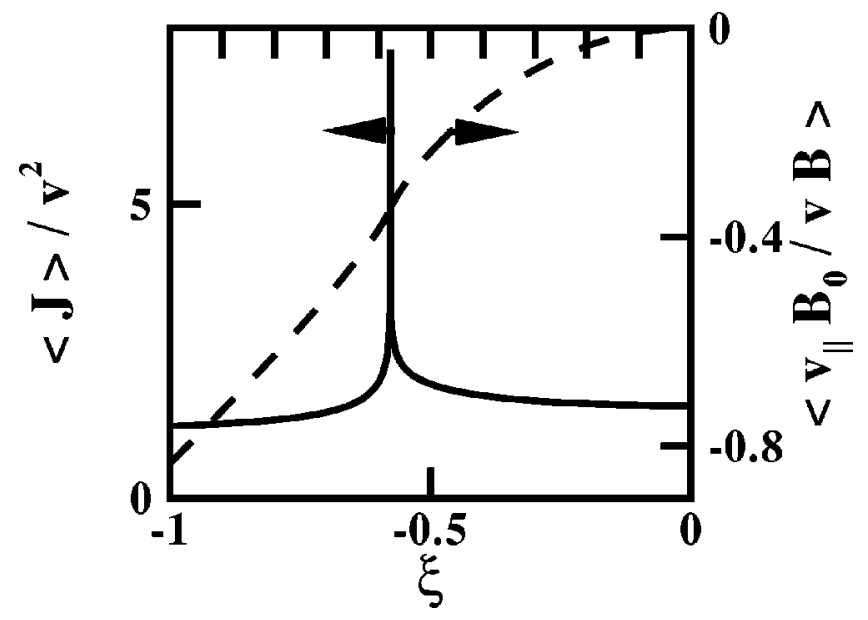

FIG. 1. Bounce averaged functions used in generating the eigenfunctions for $B=B_{0}(1+\epsilon) /\left(1+\epsilon \cos 2 \pi l / l_{0}\right)$ with $\epsilon=0.20$ and $\xi_{t}=-0.58$.

$$
\begin{array}{r}
v \xi \frac{\partial F^{p}}{\partial L}-\bar{\nu} \frac{\partial}{\partial \xi}\left(\frac{1-\xi^{2}}{\xi}\left\langle\frac{v_{\|} B_{0}}{v B}\right\rangle\right) \frac{\partial F^{p}}{\partial \xi}=-v \xi \frac{\partial f}{\partial L}, \\
|\xi| \geqslant \xi_{t},
\end{array}
$$

for the passing electron distribution, $F^{p}$. Here $\xi_{t}$ $= \pm \sqrt{1-\left(B_{0} / B\right)}$ and

$$
\left\langle\frac{v_{\|} B_{0}}{v B}\right\rangle=\frac{\int_{-l_{\max }}^{l_{\max }} d l\left(v_{\|} B_{0} / v B\right)}{\int_{-l_{\max }}^{l_{\max }} d l},
$$

for passing particles, for example. Note that Eq. (24) is complicated by the fact that it is not separable in the pitch-angle variable due the free-streaming term. This is in contrast to the analogous equation of equilibrium neoclassical transport theory. 3,4

A common approach to solving Eqs. (23) and (24) is to ignore collisions except near the trapped/passing boundary where they dominate for times short compared to the collision time. The collision operator may then be simplified and integrated directly in the boundary layer and the solution matched to that of the outer region where collisions are ignored. ${ }^{9}$ Such an approach is not appropriate, however, for time scales longer than the collision time where the effect of the Lorentz operator is felt over all of pitch-angle space. Such is the case when deriving a heat flux closure for electrons confined by a slowly evolving magnetic field. In the spirit of retaining as much of the physics of the Lorentz operator as possible, we solve Eqs. (23) and (24) by seeking solutions of the following eigenvalue equation: ${ }^{10}$

$$
\frac{\partial}{\partial \xi} \frac{1-\xi^{2}}{\xi}\left\langle\frac{v_{\|} B_{0}}{v B}\right\rangle \frac{\partial C_{n}}{\partial \xi}+\lambda_{n}\left(\frac{v \xi B}{v_{\|} B_{0}}\right) C_{n}=0 .
$$

The weight function, $\left\langle v \xi B / v_{\|} B_{0}\right\rangle=\left\langle J / v^{2}\right\rangle=\langle\nabla v \cdot \nabla \gamma$ $\left.\times \nabla \xi / v^{2}\right\rangle$, ensures orthogonality among the eigenfunctions $C_{n}$ with respect to the Jacobian, $J$, for the chosen guiding center variables, $v, \xi$ and the gyroangle, $\gamma$. The bounceaveraged quantities in Eq. (26) are shown in Fig. 1 for $B$ $=B_{0}(1+\epsilon) /\left(1+\epsilon \cos \left(2 \pi l / l_{0}\right)\right)$ with $\epsilon=0.20$ and $\xi_{t}=-0.58$. 


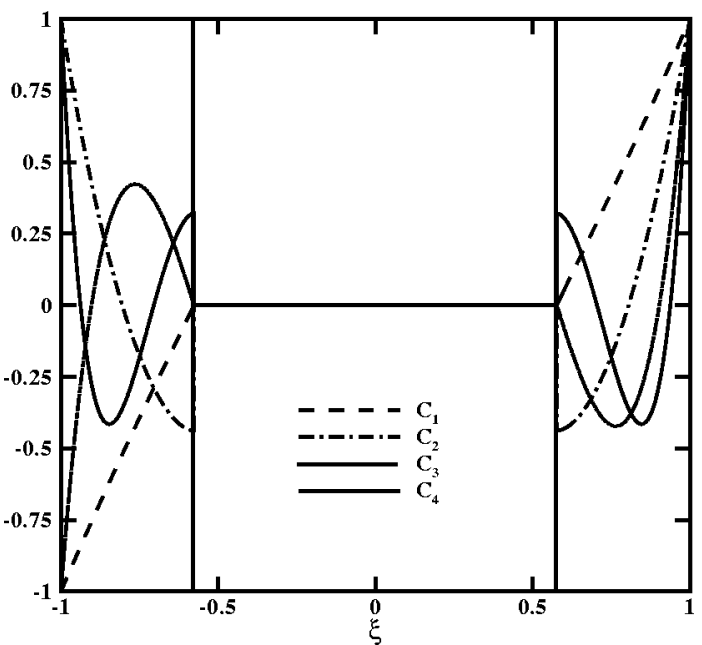

FIG. 2. Eigenfunctions for $B=B_{0}(1+\epsilon) /\left(1+\epsilon \cos \left(2 \pi l / l_{0}\right)\right)$ with $\epsilon=0.20$ and $\xi_{t}=-0.58$.

For trapped electrons, $\lambda_{n}=0$ and the eigenvalue equation may be integrated directly to yield boundary conditions for the $C_{n}$ at $\xi= \pm \xi_{t}$, namely,

$$
\frac{\partial C_{n}}{\partial \xi}=\frac{c \xi}{\left(1-\xi^{2}\right)}\left\langle\frac{v_{\|} B_{0}}{v B}\right\rangle^{-1}
$$

where $c$ is a constant. The oddness of $\partial C_{n} / \partial \xi$ about $\xi=0$ implies that $C_{n}$ is even in trapped space. We infer from this that the odd basis functions needed to represent $F^{p}$ go to zero at $\xi= \pm \xi_{t}$. Furthermore, it can be shown that the ratio $\xi /\left\langle v_{\|} B_{0} / v B\right\rangle$ diverges as $|\xi| \rightarrow 0$; hence it is necessary that the constant $c=0$ in Eq. (27) if $\partial C_{n} / \partial \xi$ is to remain bounded. The necessary result is that $\partial C_{n} / \partial \xi=0$ for $-\xi_{t}$ $\leqslant \xi \leqslant \xi_{t}$. In reality, this result does not hold near $\xi= \pm \xi_{t}$, where the bounce average operation breaks down. Previous authors have shown that a proper boundary layer theory provides corrections of order, $O\left(\sqrt{\nu / \mathbf{v}_{\|} \cdot \nabla_{l}}\right) \cdot{ }^{11}$ These corrections will be ignored in this work; hence the boundary conditions applied to $C_{n}$ at $\xi= \pm \xi_{t}$ are

$$
C_{n}=0, \text { for } n \text { odd }
$$

and

$$
\frac{\partial C_{n}}{\partial \xi}=0, \text { for } n \text { even. }
$$

Note that these are the same boundary conditions applied at the origin when generating the odd and even Legendre polynomials which may be generated from the infinite-aspectratio limit of Eq. (26).

As examples, Fig. 2 shows eigenfunctions pertaining to sinusoidal variations of the form $B=B_{0}(1+\epsilon) /(1$ $\left.+\epsilon \cos \left(2 \pi l / l_{0}\right)\right)$ with $\epsilon=0.20$ and $\xi_{t}=-0.58$ and Fig. 3 shows them for $\epsilon=0.40$ and $\xi_{t}=-0.76$. The eigenfunctions were generated using a shooting method that matched the boundary conditions at $\pm \xi_{t}$ by guessing the eigenvalue and integrating in from the exterior boundaries, $\xi= \pm 1$. The selfadjoint eigenvalue problem for the $\xi$ dependence in passing space and the requirement that $F^{t}=0$ provide for vanishing $n$

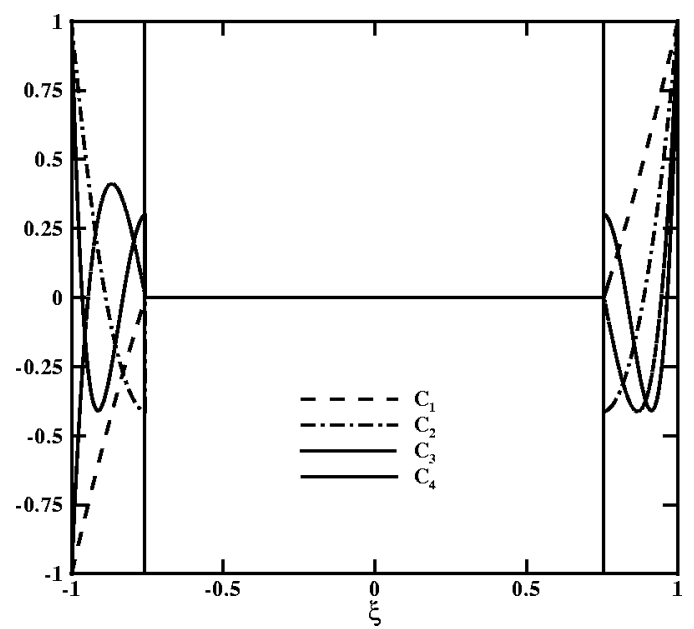

FIG. 3. Eigenfunctions for $B=B_{0}(1+\epsilon) /\left(1+\epsilon \cos \left(2 \pi l / l_{0}\right)\right)$ with $\epsilon=0.40$ and $\xi_{t}=-0.76$.

and $T$ moments of the kinetic distortion, $F$, an essential aspect of Chapman-Enskog theory [see the discussion surrounding Eqs. (13) and (14)].

We now expand $F^{p}$ in Eq. (24) in a finite set of pitchangle eigenfunctions, $F^{p}=\sum_{n=1}^{N} F_{n}(v, L) C_{n}(\xi)$, and write $\mathbf{F}=\left[F_{1}, F_{2}, \ldots, F_{N}\right]$ as a column vector of coefficients. Inserting the expanded form for $F^{p}$ in Eq. (24), multiplying by $C_{n^{\prime}}$ and integrating over the variable $\xi$ yields the following system of equations:

$$
\mathbf{I F}+\frac{v}{\bar{\nu}} \mathbf{A} \frac{\partial \mathbf{F}}{\partial L}=-\frac{v}{\bar{\nu}} \mathbf{G} \frac{\partial f}{\partial L},
$$

where the speed dependence $v / \bar{\nu}$ is stated explicitly. Here $\mathbf{I}$ is the identity matrix, A contains the ratios of the freestreaming coupling between the different eigenfunctions to the $n$th eigenvalue, and $\mathbf{G}$ is the projection of the Maxwellian drives onto the eigenfunctions.

Equation (30) is solved in the next section in order to calculate an approximate parallel heat flux for transit and collisional times long compared to the bounce time.

\section{GENERALIZED HEAT FLUX}

In this section a general heat flux is constructed and written in forms resembling that of Ref. 1. The solution of the set of equations in Eq. (30) is straightforward. The eigenvectors and associated eigenvalues of the matrix $\mathbf{A}$ are first determined. Denoting $\mathbf{W}$ as the matrix containing the eigenvectors of $\mathbf{A}$ as columns, we can expand the vector of expansion coefficients as follows: $\mathbf{F}=\mathbf{W h}$. Inserting this form for $\mathbf{F}$ into Eq. (30) and multiplying through by $\mathbf{W}^{-1}$ diagonalizes the system. The result is a set of first-order ordinary differential equations (ODEs) for the elements of $\mathbf{h}$, namely,

$$
\gamma_{i} \frac{v}{\bar{\nu}} \frac{\partial h_{i}}{\partial L}+h_{i}=-\frac{v}{\bar{\nu}} g_{i} \frac{\partial f}{\partial L},
$$

where $g_{i}$ is the $i$ th element of $\mathbf{W}^{-1} \mathbf{G}$ and $\gamma_{i}$ is the eigenvalue associated with the $i$ th column of $\mathbf{W}$. In analogy with Ref. 1, we define effective inverse collision lengths of the form $\bar{k}_{i}$ $\equiv \bar{\nu} /\left(\gamma_{i} v\right)$. 
The domain in $L$ is chosen to be $(-\infty, \infty)$. The requirement of boundedness at $\pm \infty$ demands that the homogeneous solution to Eq. (31) be set to zero. The particular solution for the passing part of the distribution function is,

$$
\begin{aligned}
F^{p}\left(L^{\prime}\right)= & -\sum_{n=1}^{N} C_{n}(\xi)\left[\sum_{\bar{k}_{i}>0} \frac{W_{n i} g_{i}}{\gamma_{i}} \int_{L^{\prime}}^{\infty} d L \frac{\partial f}{\partial L} e^{-\bar{k}_{i}\left(L-L^{\prime}\right)}\right. \\
& \left.+\sum_{\bar{k}_{i}<0} \frac{W_{n i} g_{i}}{\gamma_{i}} \int_{-\infty}^{L^{\prime}} d L \frac{\partial f}{\partial L} e^{-\bar{k}_{i}\left(L-L^{\prime}\right)}\right],
\end{aligned}
$$

where the $\bar{k}_{i}$ come in positive/negative pairs and the limits of integration are $L^{\prime}$ to $\infty$ for $\bar{k}_{i}>0$ and $-\infty$ to $L^{\prime}$ for $\bar{k}_{i}<0$. The Landau damping and collisional effects present in the $\bar{k}_{i}$ 's serve to truncate these integrals. The integral nature of $F^{p}$ results because we have allowed for arbitrary collisionality and necessarily $F^{p}$ is a function of the Maxwellian drives all along a magnetic field line. The form for $F^{p}$ may be simplified since the effective inverse collision lengths arise in positive and negative pairs, $\pm \bar{k}_{i}$. Integration by parts yields the simple form,

$$
\begin{aligned}
F^{p}\left(L^{\prime}\right)= & \sum_{n=1}^{N} C_{n}(\xi) \sum_{i=1}^{N / 2}\left|\frac{W_{n i} g_{i} \bar{k}_{i}}{\gamma_{i}}\right| \int_{L^{\prime}}^{\infty} d L[f(-L) \\
& -f(L)] e^{-\left|\bar{k}_{i}\right|\left(L-L^{\prime}\right)} .
\end{aligned}
$$

In the case of a homogeneous magnetic field, Ref. 1 used the following definition of the parallel heat flow:

$q_{\|}\left(L^{\prime}\right)=-T^{\mathrm{eq}} \int d^{3} v L_{1}^{(3 / 2)} v_{\|} F=T^{\mathrm{eq}} \int d^{3} v\left(\frac{v^{2}}{v_{\mathrm{th}}^{2}}-\frac{5}{2}\right) v \xi F$.

Analogously, we define an averaged $q_{\|}$moment in the inhomogeneous case as

$$
q_{\|}\left(L^{\prime}\right)=T^{\mathrm{eq}} \int_{l_{\min }}^{l_{\max }} d l \int d^{3} v\left(\frac{v^{2}}{v_{\mathrm{th}}^{2}}-\frac{5}{2}\right) v \xi F^{p} / \int_{l_{\min }}^{l_{\max }} d l .
$$

Orthogonality among the even and odd $C_{n}$ implies that $q_{\|}$ may be written simply as

$$
\begin{aligned}
q_{\|}\left(L^{\prime}\right)= & T^{\mathrm{eq}} \int\left\langle d^{3} v\right\rangle v L_{1}^{(3 / 2)} \xi \sum_{n \text { odd }} C_{n} \sum_{i=1}^{N / 2}\left|\frac{W_{n i} g_{i} \bar{k}_{i}}{\gamma_{i}}\right| \\
& \times \int_{L^{\prime}}^{\infty} d L[f(-L)-f(L)] e^{-\left|\bar{k}_{i}\right|\left(L-L^{\prime}\right)} .
\end{aligned}
$$

The Maxwellian drive in the heat flow integral is

$$
f(L)=\left[L_{0}^{(1 / 2)} \frac{p(L)}{p^{\mathrm{eq}}}+L_{1}^{(3 / 2)} \frac{T(L)}{T^{\mathrm{eq}}}\right] f^{\mathrm{eq}} .
$$

As in Ref. 1, the pressure drive will be ignored here. In traditional neoclassical transport theory, the $p$ drive vanishes due to the orthogonality of Laguerre polynomials. Here the additional $v$ dependence in the $\bar{k}_{i}$ 's spoils this orthogonality. We note further, that in a more complete Chapman-Enskog theory that rewrites the drives using the lowest-order moment equations, the pressure drive is absent.
In order to write a final form for $q_{\|}$that is reminiscent of the form in Ref. 1, we interchange the order of the velocity space and field line integrations and define the kernel $K\left(L^{\prime}, L\right)$ as

$$
\begin{aligned}
K\left(L^{\prime}, L\right)= & \frac{1}{v_{\text {th }}^{3}} \int\left\langle d^{3} v\right\rangle v\left(L_{1}^{(3 / 2)}\right)^{2} \xi \\
& \times \sum_{n \text { odd }} C_{n} \sum_{i=1}^{N / 2} a_{i} e^{-\left|\bar{k}_{i}\right|\left(L-L^{\prime}\right)},
\end{aligned}
$$

where $a_{i}=\left|W_{1 i} g_{i} \bar{k}_{i} / \gamma_{i}\right|$, such that

$$
q_{\|}=\frac{n^{\mathrm{eq}} v_{\mathrm{th}}}{\pi^{3 / 2}} \int_{L^{\prime}}^{\infty} d L[T(-L)-T(+L)] K\left(L^{\prime}, L\right) .
$$

Here $K\left(L^{\prime}, L\right)$ contains the collisional and trapping information of a distribution of electrons as they scatter in pitch angle off a background Maxwellian plasma. The similar form of this general expression for the parallel heat flow with previous collisionless expressions ${ }^{12,13}$ was emphasized in Ref. 1. It was also shown there that Eq. (39) reduces to the familiar form, $q_{\|} \sim \kappa_{\|} \nabla_{\|} T$, in the collisional limit. ${ }^{14}$ It is important to note, however, that here the parallel conductivity, $\kappa_{\|}$, is reduced by particle trapping and collisions near the trapped/ passing boundary. Furthermore, the nearly collisionless limit of Eq. (39) contains important corrections due to particle trapping that were absent in previous collisionless expressions for the parallel heat flow. These effects are highlighted in the next section.

\section{SAMPLE CALCULATION}

In this section, we return to the case of sinusoidal magnetic wells and explore the resultant parallel heat flow for sinusoidal temperature perturbations of scale length, $L_{T}$ $\gg l_{0}$, namely,

$$
T(L)=T^{\mathrm{eq}}+\widetilde{T} \sin \left(\frac{2 \pi L}{L_{T}}\right) .
$$

Recall that the eigenfunctions for the case of $B=B_{0}(1$ $+\epsilon) /\left(1+\epsilon \cos \left(2 \pi l / l_{0}\right)\right)$ with $\epsilon=0.2$ and $\epsilon=0.4$ were presented in Figs. 2 and 3. The orderings $L_{T} \sim L_{\nu} \gg l_{0}$, where $L_{\nu}$ is the characteristic collision length, permit a study of the parallel heat flow response as collisionality is varied. Specifically, we will choose representative scale lengths for a helical magnetic island in an axisymmetric tokamak. If the length of magnetic wells is $1 \mathrm{~m}$, then we require $L_{T} \sim L_{\nu}$ $\gg 1 \mathrm{~m}$ for the expression in Eq. (39) to be valid. For $1 \mathrm{keV}$ plasmas $L_{\nu} \approx 100 \mathrm{~m}$; hence we let $L_{T}$ vary from $10 \mathrm{~m}$, which is representative of temperature gradient scale lengths inside magnetic islands, to $L_{T} \rightarrow \infty$, which is relevant near the $\mathrm{X}$ and O-points of a magnetic island.

In this demonstration, it is necessary to pick the number of basis functions in the pitch-angle basis function expansion in order to preserve the ordering $\nu \ll \mathbf{v}_{\|} \cdot \nabla_{l}$. The reason for 


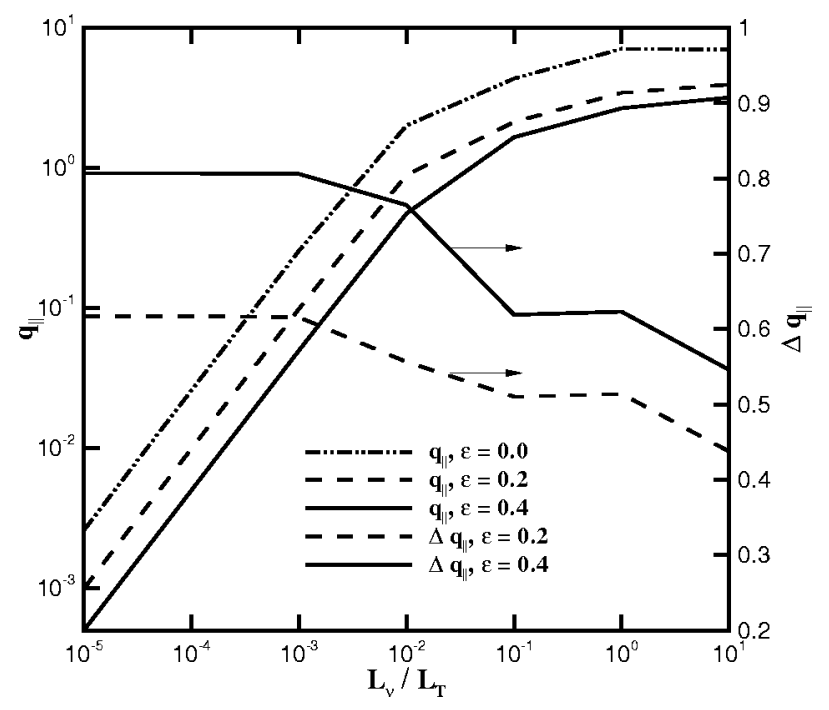

FIG. 4. Parallel heat flow response $q_{\|}$for homogeneous and inhomogeneous $|B|$ and enhanced reduction $\Delta q_{\|}$for $T(L)=T^{\mathrm{eq}}+\widetilde{T} \sin \left(2 \pi L / L_{T}\right)$ show the effect of trapped particles at all ratios of $L_{\nu} / L_{T}$ and the enhanced reduction due to collisions in regimes with $L_{\nu} / L_{T} \leqslant 1$. Here $Z_{\text {eff }}=1$ and $q_{\|}$and $\Delta q_{\|}$are normalized to $n^{\mathrm{eq}} v_{\text {the }} \widetilde{T} / \pi^{3 / 2}$.

this is that the eigenvalues of the basis functions enter into the ordering in the following manner, $\lambda_{n} \ll L_{\nu} / l_{0}$. Care must be taken to choose enough basis functions in regimes of moderate to low collisionality to resolve the pitch-angle dependence of the distribution function, but not too many that the ordering which permits a bounce-average, namely, $\lambda_{n}$ $\ll L_{\nu} / l_{0}$, is invalidated. For the results presented below, two basis functions were used to resolve the pitch-angle dependence of the kinetic distortion for $L_{\nu} / L_{T} \leqslant 0.1$ and four were used for $L_{\nu} / L_{T} \geqslant 1.0$

Figure 4 compares the heat flow at $L^{\prime}=0$ (left $y$-axis) for the cases of uniform and nonuniform magnetic fields. As the ratio of $L_{\nu} / L_{T}$ increases, the heat flow increases because electrons are more mobile and carry more heat. In the collisional limit $\left(L_{\nu} / L_{T}<0.01\right)$, the heat flow is proportional to the local temperature gradient as in the classical Braginskii analysis. ${ }^{14}$ It is important to note, however, that in cases with an inhomogeneous magnetic field, we still require that the bounce time be short compared to the collision time. That is, we are exploring the effect of varying collisionality while always remaining in the familiar banana transport regime. In collisional to moderately collisional regimes $\left(L_{\nu} / L_{T}<1.0\right)$, the reduction in heat flow for the cases $\epsilon=0.2$ and $\epsilon=0.4$ is due to the combined effect of trapped electrons, which carry no heat over longer gradient scale lengths, and enhanced collisional effects near the trapped/passing boundary. In the nearly collisionless limit, the reduction in heat flow is due solely to trapped particles.

Figure 4 also shows the fractional reduction (right $y$-axis) in heat flow between the inhomogeneous cases and homogeneous case as collisionality is varied. At $L_{\nu} / L_{T}$ $\leqslant 10$, the reduction approaches the trapped particle fraction namely, $35 \%$ for $\epsilon=0.2$ and $50 \%$ for $\epsilon=0.4$. The enhanced collisionality between trapped and passing electrons is shown in the regime $L_{\nu} / L_{T}<1.0$, where the larger reduction in the case of $\epsilon=0.4$ as compared to $\epsilon=0.2$ is evident.

\section{CONCLUSIONS}

In this paper, a variation of neoclassical transport theory was used to derive the parallel electron heat flow closure in Eq. (39). This closure allows for multiple scale lengths parallel to the magnetic field including short scale magnetic mirroring and long scale electron temperature gradients. For times long compared to the bounce time, it is possible to preserve the maximal ordering between free-streaming and bounce-averaged pitch-angle scattering effects via an expansion in pitch-angle eigenfunctions. This maximal ordering leads to an integral form for the parallel heat flow that depends on temperature variations all along a magnetic field line. The sample heat flow calculation in Sec. IV showed that in nearly collisionless plasmas the heat flow is reduced by the trapped electron fraction. Furthermore, in collisional to moderately collisional plasmas, the heat flow is reduced both by trapped electrons and by the enhanced collisionality between trapped and passing electrons (see Fig. 4). The results of this demonstration attest to the fact that the closure scheme outlined in this work provides a quantitative means for calculating parallel electron heat flow along inhomogeneous magnetic fields in simulations of high-performance toroidal fusion experiments.

\section{ACKNOWLEDGMENTS}

This research has been supported by the DOE under Grant Nos. DE-FG03-01ER54618 and DE-FG0286ER53218.

${ }^{1}$ E. D. Held, J. D. Callen, C. C. Hegna, and C. R. Sovinec, Phys. Plasmas 8, 1171 (2001).

${ }^{2}$ B. D. G. Chandran and S. C. Cowley, Phys. Rev. Lett. 80, 3077 (1998).

${ }^{3}$ F. L. Hinton and R. D. Hazeltine, Rev. Mod. Phys. 48, 239 (1976).

${ }^{4}$ S. P. Hirshman and D. J. Sigmar, Nucl. Fusion 21, 1079 (1981).

${ }^{5}$ R. D. Hazeltine, Plasma Phys. 15, 77 (1973).

${ }^{6} \mathrm{~S}$. Chapman and T. G. Cowling, The Mathematical Theory of Nonuniform Gases (Cambridge University Press, Cambridge, 1939).

${ }^{7}$ Z. Chang and J. D. Callen, Phys. Fluids B 4, 1167 (1992).

${ }^{8}$ S. Chandrasekhar, Rev. Mod. Phys. 15, 1 (1943).

${ }^{9}$ R. C. Morris, M. G. Haines, and R. J. Hastie, Phys. Plasmas 3, 4513 (1996).

${ }^{10}$ J. G. Cordey, Nucl. Fusion 16, 499 (1976).

${ }^{11}$ F. Hinton and M. N. Rosenbluth, Phys. Fluids 16, 836 (1973).

${ }^{12}$ G. W. Hammett and F. W. Perkins, Phys. Rev. Lett. 64, 3019 (1990).

${ }^{13}$ P. B. Snyder, G. W. Hammet, and W. Dorland, Phys. Plasmas 4, 3974 (1997).

${ }^{14}$ S. I. Braginskii, Transport Processes in a Plasma, edited by M. A. Leontovich (Consultants Bureau, New York, 1965), Vol. 1. 